DOI 10.4171/JEMS/338

Jacek Brodzki · Graham A. Niblo · Nick Wright

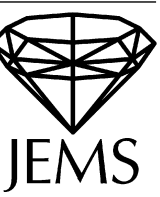

\title{
Pairings, duality, amenability and bounded cohomology
}

Received March 23, 2010 and in revised form August 25, 2010

\begin{abstract}
We give a new perspective on the homological characterisations of amenability given by Johnson \& Ringrose in the context of bounded cohomology and by Block \& Weinberger in the context of uniformly finite homology. We examine the interaction between their theories and explain the relationship between these characterisations. We apply these ideas to give a new proof of non-vanishing for the bounded cohomology of a free group.
\end{abstract}

We will illuminate the relationship between the following two remarkable characterisations of amenability for a group.

Theorem (Johnson-Ringrose, [5]). A group $G$ is amenable if and only if

$$
H_{b}^{1}\left(G,\left(\ell^{\infty}(G) / \mathbb{C}\right)^{*}\right)=0 .
$$

Theorem (Block-Weinberger, [1]). A group $G$ is amenable if and only if $H_{0}^{\mathrm{uf}}(G) \neq 0$.

It should be noted that both statements are part of a much larger picture. In the first theorem $H_{b}^{1}\left(G,\left(\ell^{\infty}(G) / \mathbb{C}\right)^{*}\right)$ denotes the bounded cohomology with coefficients in the dual of $\ell^{\infty}(G) / \mathbb{C}$. Vanishing of the cohomology is guaranteed by the triviality of a particular cocycle, the Johnson class $[J]$, defined below. Furthermore this ensures triviality of bounded cohomology with any coefficients in dimensions greater than or equal to 1 . In the second theorem $H_{0}^{\text {uf }}(G)$ denotes the uniformly finite homology of Block and Weinberger. Vanishing of the zero-dimensional homology group is guaranteed by the triviality of a fundamental class. (Analogous results concerning $\ell^{p}$ homology and $c_{0}$ homology were considered by Elek in [4] who introduced the attractive technology of binary schemes as a generalisation of the Block-Weinberger method to kill 0-cycles in a non-amenable graph. We are grateful to the referee for drawing our attention to this paper.)

It should also be noted that the notion of amenability and the definition of uniformly finite homology can be extended from groups to arbitrary metric spaces, and the BlockWeinberger theorem applies in full generality. However there is no natural analog for the Johnson and Ringrose theorem in that context. We examine this issue further in [2] where we define a cohomology (analogous to bounded cohomology) for a metric space. We

J. Brodzki, G. A. Niblo, N. Wright: School of Mathematics, University of Southampton, Highfield, Southampton, SO17 1SH, England;

e-mail: J.Brodzki@ soton.ac.uk, G.A.Niblo@ soton.ac.uk, N.J.Wright@soton.ac.uk 
use it there to give a generalisation of the Johnson-Ringrose theorem characterising Yu's property $\mathrm{A}$, which is the natural generalisation of amenability in the context of coarse geometry.

Here we give a short, unified proof of the Johnson-Ringrose and Block-Weinberger theorems by exploiting duality and the short exact sequence of coefficients

$$
0 \rightarrow \mathbb{C} \stackrel{\iota}{\rightarrow} \ell^{\infty}(G) \stackrel{\pi}{\rightarrow} \ell^{\infty}(G) / \mathbb{C} \rightarrow 0 .
$$

The interaction between the two theories arises from a pairing between homology and cohomology.

It is well known that the free group of rank at least 2 is non-amenable and therefore according to Johnson and Ringrose the class $[J]$ is non-zero. As an application of the duality principle we demonstrate this non-vanishing by pairing $J$ with an explicit $\ell^{1}$-cycle. This construction should be compared with the argument in [6] for the linear independence of the Brooks cocycles in the bounded cohomology of a surface.

Recall the following definition.

Definition. A mean on a group $G$ is a positive linear functional $\mu$ on $\ell^{\infty}(G)$ such that $\|\mu\|=1$. A group $G$ is amenable if it admits a $G$-invariant mean.

Recall that for a Banach space $V$ equipped with an isometric action of a group $G$, $C_{b}^{m}\left(G, V^{*}\right)$ denotes the $G$-module of equivariant bounded cochains $\phi: G^{m+1} \rightarrow V^{*}$. (Here bounded is defined by the Banach norm on the dual space $V^{*}$.) This yields a cochain complex $\left(C_{b}^{m}\left(G, V^{*}\right), d\right)$ where $d$ denotes the natural differential induced from the homogeneous bar resolution:

$$
d \phi\left(g_{0}, \ldots, g_{m+1}\right)=\sum_{i=0}^{m+1}(-1)^{i} \phi\left(g_{0}, \ldots, \hat{g}_{i}, \ldots, g_{m+1}\right) .
$$

(As usual $\hat{g}_{i}$ denotes the omission of the entry indexed $i$.)

The cohomology of this complex is the bounded cohomology of the group with coefficients in $V^{*}$, denoted $H_{b}^{*}\left(G, V^{*}\right)$. For $V=\ell^{\infty}(G) / \mathbb{C}$ there is a particular class in dimension 1 detecting amenability which we will call the Johnson element. This is represented by the function

$$
J\left(g_{0}, g_{1}\right)=\delta_{g_{1}}-\delta_{g_{0}},
$$

where $\delta_{g}$ denotes the Dirac delta function supported at $g$. Note that $J\left(g_{0}, g_{1}\right)$ lies in the predual $\ell_{0}^{1}(G)$ of $V$, which we view as a subspace of $V^{*}$.

Now consider the chain complex $\left(C_{m}^{\ell^{1}}(G, V), \partial\right)$, where $C_{m}^{\ell^{1}}(G, V)$ consists of equivariant functions $c: G^{m+1} \rightarrow V$ which are $\ell^{1}$ on the subspace $\{e\} \times G^{m}$. The boundary map is defined by

$$
\partial c\left(g_{0}, \ldots, g_{m-1}\right)=\sum_{g \in G, i \in\{0, \ldots, m\}}(-1)^{i} c\left(g_{0}, \ldots, g_{i-1}, g, g_{i}, \ldots, g_{m-1}\right) .
$$

The homology of this complex is the $\ell^{1}$-homology of the group with coefficients in $V$, denoted $H_{*}^{\ell^{1}}(G, V)$. 
We note that the pairing of $V^{*}$ with $V$, denoted $\langle-,-\rangle_{V}$, induces a pairing of $H_{b}^{m}\left(G, V^{*}\right)$ with $H_{m}^{\ell^{1}}(G, V)$ defined by

$$
\langle[\phi],[c]\rangle=\sum_{g_{1}, \ldots, g_{m} \in G}\left\langle\phi\left(e, g_{1}, \ldots, g_{m}\right), c\left(e, g_{1}, \ldots, g_{m}\right)\right\rangle_{V} .
$$

It is clear that the pairing is defined at the level of cochains. It is well defined on classes since the differential $d$ is the adjoint of the boundary map $\partial$.

The proof of the following result is a standard application of the snake lemma:

Proposition. The short exact sequence of $G$-modules $0 \rightarrow \mathbb{C} \stackrel{\iota}{\rightarrow} \ell^{\infty}(G) \stackrel{\pi}{\rightarrow} \ell^{\infty}(G) / \mathbb{C}$ $\rightarrow 0$ induces a short exact sequence of chain complexes

$$
0 \rightarrow C_{m}^{\ell^{1}}(G, \mathbb{C}) \stackrel{\iota}{\rightarrow} C_{m}^{\ell^{1}}\left(G, \ell^{\infty}(G)\right) \stackrel{\pi}{\rightarrow} C_{m}^{\ell^{1}}\left(G, \ell^{\infty}(G) / \mathbb{C}\right) \rightarrow 0
$$

and hence a long exact sequence of $\ell^{1}$-homology groups.

The short exact sequence of $G$-modules $0 \rightarrow\left(\ell^{\infty}(G) / \mathbb{C}\right)^{*} \stackrel{\pi^{*}}{\rightarrow} \ell^{\infty}(G)^{*} \stackrel{\iota^{*}}{\rightarrow} \mathbb{C} \rightarrow 0$ induces a short exact sequence of cochain complexes

$$
0 \rightarrow C_{b}^{m}\left(G,\left(\ell^{\infty}(G) / \mathbb{C}\right)^{*}\right) \stackrel{\pi^{*}}{\rightarrow} C_{b}^{m}\left(G, \ell^{\infty}(G)^{*}\right) \stackrel{\iota^{*}}{\rightarrow} C_{b}^{m}(G, \mathbb{C}) \rightarrow 0
$$

and hence a long exact sequence of bounded cohomology groups.

Now we consider the Block-Weinberger uniformly finite homology. The definition in [1] works for an arbitrary metric space, however we will restrict attention to the case of a countable discrete group $G$, where the group is equipped with a proper left invariant metric $d$, which simplifies the definition.

Let $C_{q}^{\mathrm{uf}}(G, \mathbb{C})$ denote the vector space of complex valued functions $c: G^{q+1} \rightarrow \mathbb{C}$ which are bounded, and have controlled support in the sense that there is a constant $K$ (depending on the function $c$ ) such that if $\operatorname{diam}\left\{g_{0}, \ldots, g_{q}\right\} \geq K$ then $c\left(g_{0}, \ldots, g_{q}\right)=0$. Again the boundary map $\partial$ on the homogeneous bar resolution induces the differential $\partial: C_{q}^{\mathrm{uf}}(G, \mathbb{C}) \rightarrow C_{q-1}^{\mathrm{uf}}(G, \mathbb{C})$. The uniformly finite homology of $G$ is the homology of this chain complex. Let 1 denote the constant function with value $1 \in \mathbb{C}$. Block and Weinberger showed that the fundamental class [1] in degree 0 is non-trivial if and only if $G$ is amenable [1, Theorem 3.1].

In fact the uniformly finite homology coincides with the classical group homology $H_{q}\left(G, \ell^{\infty}(G)\right)$, with coefficients in the module of bounded complex valued functions on $G$. The corresponding chain complex consists of equivariant functions from $G^{q+1}$ to $\ell^{\infty}(G)$ which are supported on finitely many $G$-orbits. To see that the two homologies coincide we note that a cochain $c \in C_{q}^{\mathrm{uf}}(G, \mathbb{C})$ can be inflated to a map $\bar{c}: G^{q+1} \rightarrow \ell^{\infty}(G)$ by setting $\bar{c}\left(g_{0}, \ldots, g_{q}\right)(g)=c\left(g^{-1} g_{0}, \ldots, g^{-1} g_{q}\right)$. This function is, by construction, equivariant and the controlled support condition ensures that $\bar{c}$ is supported on finitely many $G$-orbits. It is easy to see that this process is invertible. Since the differentials in both complexes are induced from the homogeneous bar resolution, this map is an isomorphism 
between the chain complexes, hence we may identify $H_{q}^{\text {uf }}(G, \mathbb{C})$ with $H_{q}\left(G, \ell^{\infty}(G)\right)$. We note that $\overline{\mathbf{1}}=\iota \mathbf{1}$.

The function 1 represents classes in all of the following objects: $H_{b}^{0}(G, \mathbb{C}), H_{0}(G, \mathbb{C})$, $H_{0}^{\ell^{1}}(G, \mathbb{C})$. The above identification shows that the Block-Weinberger fundamental class is $\iota[1] \in H_{0}\left(G, \ell^{\infty}(G)\right)$. The Johnson class is $d[\mathbf{1}] \in H_{b}^{1}\left(G,\left(\ell^{\infty}(G) / \mathbb{C}\right)^{*}\right)$, where $d$ denotes the connecting map $H_{b}^{0}(G, \mathbb{C}) \rightarrow H_{b}^{1}\left(G,\left(\ell^{\infty}(G) / \mathbb{C}\right)^{*}\right)$. To see this, note that $d[\mathbf{1}]$ is obtained by lifting 1 to the element $g \mapsto \delta_{g}$ in $C_{b}^{0}\left(G,\left(\ell^{\infty}(G)\right)^{*}\right)$ and taking the coboundary. This produces the Johnson cocycle $J\left(g_{0}, g_{1}\right)=\delta_{g_{1}}-\delta_{g_{0}}$. By exploiting the connecting maps arising in the proposition together with these observations we will obtain a new proof that $G$ is amenable if and only if the Johnson cocycle in bounded cohomology vanishes, and that this is equivalent to non-vanishing of the Block-Weinberger fundamental class.

We now turn to the statement and proof of our main theorem. We first observe that the non-vanishing of $H_{b}^{0}\left(G, \ell^{\infty}(G)^{*}\right)$ is equivalent to amenability since elements of $H_{b}^{0}\left(G, \ell^{\infty}(G)^{*}\right)$ are maps $\phi: G \rightarrow \ell^{\infty}(G)^{*}$ which are $G$-equivariant and also, since they are cocycles, constant on $G$. Hence the value of a cocycle $\phi$ at any (and hence all) $g \in G$ is a $G$-invariant linear functional on $\ell^{\infty}(G)$. If $\phi$ is non-zero then taking its absolute value and normalising we obtain an invariant mean on the group. Conversely, any invariant mean on the group is an invariant linear functional on $\ell^{\infty}(G)$ and hence gives a non-zero element of $H_{b}^{0}\left(G, \ell^{\infty}(G)^{*}\right)$.

Theorem 1. Let $G$ be a countable discrete group. The following are equivalent:

(1) $G$ is amenable.

(2) $\iota^{*}: H_{b}^{0}\left(G, \ell^{\infty}(G)^{*}\right) \rightarrow H_{b}^{0}(G, \mathbb{C})$ is surjective.

(3) The Johnson class $d[\mathbf{1}]$ vanishes in $H_{b}^{1}\left(G,\left(\ell^{\infty}(G) / \mathbb{C}\right)^{*}\right)$.

(4) $\langle d[1],[c]\rangle=0$ for all $[c]$ in $H_{1}^{\ell^{1}}\left(G, \ell^{\infty}(G) / \mathbb{C}\right)$. (Hence for a non-amenable group, the non-triviality of $d[\mathbf{1}]$ is detected by the pairing.)

(5) $\iota[1] \in H_{0}^{\ell^{1}}\left(G, \ell^{\infty}(G)\right)$ is non-zero.

(6) The Block-Weinberger fundamental class $\iota[1] \in H_{0}\left(G, \ell^{\infty}(G)\right)$ is non-zero.

Proof. (1) $\Rightarrow(2)$ since $H_{b}^{0}(G, \mathbb{C})=\mathbb{C}$, and for $\mu$ an invariant mean, $\iota^{*}[\mu]=[\mathbf{1}]$.

(2) $\Leftrightarrow(3)$ : By exactness, surjectivity of $\iota^{*}$ is equivalent to vanishing of $d$, hence in particular this implies $d[\mathbf{1}]=0$. The converse follows from the fact that [1] generates $H_{b}^{0}(G, \mathbb{C})$, so if $d[\mathbf{1}]=0$ then $d=0$ and $\iota^{*}$ is surjective.

The implication $(3) \Rightarrow(4)$ is trivial.

$(4) \Rightarrow(5)$ : (4) is equivalent to $\langle[\mathbf{1}], \partial[c]\rangle=0$ for all $[c]$ in $H_{1}^{\ell^{1}}\left(G, \ell^{\infty}(G) / \mathbb{C}\right)$ by duality. We note that the space of 0 -cycles in $C_{0}^{\ell^{1}}(G, \mathbb{C})$ is $\mathbb{C}$, and that the pairing of the class $[\mathbf{1}]$ in $H_{b}^{0}(G, \mathbb{C})$ with the class $[\mathbf{1}]$ in $H_{0}^{\ell^{1}}(G, \mathbb{C})$ is $\langle[\mathbf{1}],[\mathbf{1}]\rangle=1$. Hence [1] $\in$ $H_{0}^{\ell^{1}}(G, \mathbb{C})$ is not a boundary. Thus $H_{0}^{\ell^{1}}(G, \mathbb{C})=\mathbb{C}$ and the pairing with $H_{b}^{0}(G, \mathbb{C})$ is faithful so $\langle[\mathbf{1}], \partial[c]\rangle=0$ for all $[c]$ implies $\partial=0$. From this we deduce that $\iota$ is injective by exactness, hence we have (5): $\iota[1]$ is non-zero. 
$(5) \Rightarrow(6)$ since $\iota[\mathbf{1}] \in H_{0}^{\ell^{1}}\left(G, \ell^{\infty}(G)\right)$ is the image of the fundamental class in $H_{0}\left(G, \ell^{\infty}(G)\right)$ under the forgetful map.

$(6) \Rightarrow(1)$ : We use an argument due to Nowak. Let $\delta: C^{0}\left(G, \ell^{1}(G)\right) \rightarrow C^{1}\left(G, \ell^{1}(G)\right)$ denote the restriction of $d: C^{0}\left(G, \ell^{\infty}(G)^{*}\right) \rightarrow C^{1}\left(G, \ell^{\infty}(G)^{*}\right)$. This is the predual of $\partial$. First we note that $\delta$ is not bounded below, since if it were then $\partial=\delta^{*}$ would be surjective and $H_{0}\left(G, \ell^{\infty}(G)\right)$ would vanish, giving $\iota[\mathbf{1}]=0$, which is a contradiction.

The fact that $\delta$ is not bounded below is precisely the Reiter condition for amenability.

As an example of this approach we give a proof of non-amenability for a non-abelian free group $F_{n}$ by constructing an explicit element $[c] \in H_{1}^{\ell^{1}}\left(F_{n}, \ell^{\infty}\left(F_{n}\right) / \mathbb{C}\right)$ for which $\langle d[\mathbf{1}],[c]\rangle \neq 0$.

Let $F_{n}$ be a non-abelian free group with free basis $s_{1}, \ldots, s_{n}$, and let $S=$ $\left\{s_{1}^{ \pm 1}, \ldots, s_{n}^{ \pm 1}\right\}$. Let $\Gamma$ denote the Cayley graph of $F_{n}$ with respect to this generating set. $\Gamma$ is a tree and the action of $G$ on $\Gamma$ extends to the Gromov boundary. We choose a point $p$ in the Gromov boundary of $\Gamma$.

For a generator $s \in S$ we set $c(e, s)(g)=1$ if $(e, s)$ is the first edge on the geodesic from $e$ to $g p$ and set $c(e, s)(g)=0$ otherwise. Extending the definition by equivariance we obtain a function $c$ defined on the edges of $\Gamma$ with values in $\ell^{\infty}\left(F_{n}\right)$ and this represents an element $\widehat{c} \in \ell^{\infty}\left(F_{n}\right) / \mathbb{C}$.

Now consider $\partial c(e)=\sum_{s \in S} c(s, e)-c(e, s)$. For a given $g$ exactly one of the edges $(e, s), s \in S$, is the first edge on the geodesic $[e, g p]$, so the sum $\sum_{s \in S} c(e, s)$ is the constant function 1 on $F_{n}$. On the other hand for a generator $s, c(s, e)(g)=1$ if and only if the edge $(s, e)$ is the first edge on the geodesic from $s$ to $g p$. For a given $g \in F_{n}$ there is a unique $s \in S$ which lies on the geodesic from $e$ to $g p$, and this is the only one for which the corresponding term of the sum $\sum_{s \in S} c(s, e)$ takes the value 0 , so this sum is the constant function $(2 n-1) \mathbf{1}$.

Hence $\partial c(e)=(2 n-1) \mathbf{1}-\mathbf{1}=(2 n-2) \mathbf{1}$. Now by equivariance $\partial c(g)=(2 n-2) \mathbf{1}$ for all $g$, hence $\partial \widehat{c}$ vanishes in $\ell^{\infty}\left(F_{n}\right) / \mathbb{C}$, and therefore $\widehat{c}$ represents an element in $H_{1}^{\ell^{1}}\left(F_{n}, \ell^{\infty}\left(F_{n}\right) / \mathbb{C}\right)$.

We now compute the pairing $\langle d[\mathbf{1}],[\widehat{c}]\rangle$ :

$$
\langle d[\mathbf{1}],[\widehat{c}]\rangle=\langle[\mathbf{1}], \partial[\widehat{c}]\rangle=\langle[\mathbf{1}],[\partial c]\rangle=\langle[\mathbf{1}],(2 n-2)[\mathbf{1}]\rangle=2 n-2 .
$$

Hence $F_{n}$ is not amenable for $n>1$.

We conclude by noting that amenability is also equivalent to vanishing of the Johnson class as an element of the classical group cohomology $H^{1}\left(G,\left(\ell^{\infty}(G) / \mathbb{C}\right)^{*}\right)$. To see this, replace the pairing of $H_{b}^{1}\left(G,\left(\ell^{\infty}(G) / \mathbb{C}\right)^{*}\right)$ and $H_{1}^{\ell^{1}}\left(G, \ell^{\infty}(G) / \mathbb{C}\right)$ in the proof of Theorem 1 with the standard pairing of $H^{1}\left(G,\left(\ell^{\infty}(G) / \mathbb{C}\right)^{*}\right)$ and $H_{1}\left(G, \ell^{\infty}(G) / \mathbb{C}\right)$, hence deducing that vanishing of the Johnson element in $H^{1}\left(G,\left(\ell^{\infty}(G) / \mathbb{C}\right)^{*}\right)$ implies nonvanishing of the Block-Weinberger fundamental class. Hence we obtain the following theorem. 
Theorem 2. Let $G$ be a countable discrete group. The following are equivalent:

(1) $G$ is amenable.

(2) [1] lies in the image of $i^{*}: H^{0}\left(G, \ell^{\infty}(G)^{*}\right) \rightarrow H^{0}(G, \mathbb{C})$.

(3) The Johnson class $d[\mathbf{1}]$ vanishes in $H^{1}\left(G,\left(\ell^{\infty}(G) / \mathbb{C}\right)^{*}\right)$.

Acknowledgments. This research was partially supported by EPSRC grant EP/F031947/1.

\section{References}

[1] Block, J., Weinberger, S.: Aperiodic tilings, positive scalar curvature and amenability of spaces. J. Amer. Math. Soc. 5, 907-918 (1992) Zbl 0780.53031 MR 1145337

[2] Brodzki, J., Niblo, G. A., J. Wright, N.: A cohomological characterisation of Yu's property A for metric spaces. Geom. Topol. 16, 391-432 (2012)

[3] Brooks, R.: Some remarks on bounded cohomology. In: Riemann Surfaces and Related Topics (Stony Brook, NY, 1978), Ann. of Math. Stud. 97, Princeton Univ. Press, Princeton, NJ, 53-63 (1981) Zbl 0457.55002 MR 0624804

[4] Elek, G.: Amenability, $\ell^{p}$-homologies and translation invariant functionals. J. Austral. Math. Soc. Ser. A 64, 111-119 (1998) Zbl 0918.43004 MR 1646617

[5] Johnson, B. E.: Cohomology in Banach algebras. Mem. Amer. Math. Soc. 127 (1972) Zbl 0256.18014 MR 0374934

[6] Mitsumatsu, Y.: Bounded cohomology and $\ell^{1}$ homology of surfaces. Topology 23, 465-471 (1984) Zbl 0568.55002 MR 0780736

[7] Yu, G.: The coarse Baum-Connes conjecture for spaces which admit a uniform embedding into Hilbert space. Invent. Math. 139, 201-240 (2000) Zbl 0956.19004 MR 1728880 